\section{Biomedical aspects of} \section{magnetic nanoparticles}

Adam Curtis

Centre for Cell Engineering, University of Glasgow, UK

$T$ hough all the components of the body are either dia-, para, superpara- or ferromagnetic, the magnetic fields required which are likely to produce obvious effects on the body are very large. Even red blood cells with micrograms each of the iron protein haemoglobin are relatively unreactive to large fields or steep field gradients. However, there is sufficient iron present for MRI to be possible without adding iron-rich or other contrast enhancing reagents. Indeed many cell types contain magnetite or other iron oxide nanoparticles-it is not clear whether these are generated by some standard biochemical process or are adventitious particles acquired from the environment.

The other natural iron-containing compounds in the body are haemosiderin, ferritin, transferrin and the cytochromes. The question of whether there are hazards or effects from magnetic fields of the magnitudes normally encountered in the environment even in the 'built' environment acting on these molecules is unlikely though unresolved. But the electromagnetic fields produced by mobile phones may perhaps be large enough to have pathological effects (see Cranfield et al. 2003 for a suggestion as to mechanism).

However the most likely exposures to large fields or field gradients arises from therapy or diagnosis. MRI produces images of both hard and soft organs and this is useful in diagnosis and in following the course of therapy. MRI contrast reagents are often used and there are a number of potential compounds for infusion or injection. Basically there are three ways in which a contrast reagent may act. It may not enter or attach to cells and simply act as an indicator of fluid-filled space eg the knee capsule. It may attach to the outside of cells or lastly it may be taken into the cells. Closely related to these biological differences are the routes by which the agent reaches the target area. $A$ wider and wider range of effective contrast agents continue to be developed and perhaps noteworthy amongst these are dendrimer based agents to help image the lymph system (Kobayashi et al. 2003), putrescineGd-amyloid beta peptides for Alzheimer plaque detection (Poduslo et al. 2003).

There are advantages in using strongly magnetic nanoparticle contrast reagents, at least in theory, because there is a possibility that the particles can be localised in the desired region by applying local magnetic field gradients. There is also the possibility of producing localised hyperthermia when an organ loaded with nanoparticles is exposed to electromagnetic radiation. There are, however, potential if not real hazards with the use of superparamagnetic nanoparticles which are discussed below.

The localizability of the particles by applying external small fields is of apparent value in other types of therapy, for instance drug delivery or hyperthermia. It has been usual to suppose that the route or delivery would be by injection either through a vascular route or by localised injection into a tissue. After injection the particles would either remain un-attached to cells and drift around the body fluids.
If attachment was reversible particles might move from cell to cell, The next phase would be the endocytosis of the particles into the cells. If the cells remain alive the particles are very unlikely to reenter the extracellular space. The final localisation of the particles would be intracellular in many cases. Though it is clear from in vitro and some in vivo experiments that cells can survive the presence of such particles in moderate loadings for moderate periods of time, little is known about this. However, data from 'epidemological' sources suggests that carbon nanoparticles particles in the form of soot may present a risk at high exposures. On the otherhand 'natural' garden fires produce carbon nanotubes. Post-mortem samples should be taken from the lungs of people to discover if there is nanoparticle loading in the cells and if so whether it is associated with disease. Magnetite or maghemite particles may present an additional risk because the $\mathrm{pH}$ in the endo-lysosomes (see Fig 1a) is low. This final destination is so acidic that ferric ions may be formed which will damage many biochemical processes. Silica coated nanoparticles may be resistant to this effect.

Drug delivery is another perceived use of superparamagnetic particles. The drug would be attached to a surface coating on the particles such as dextran, PVA, polyglycols etc by fairly standard derivatisation chemistry. Again the application of external magnetic fields would be used to 'capture' the particles in the desired site. Besides the localizability of the particles the immobilisation of a signal molecule on the particles may protect it to an extent from degradation and the long term binding to a receptor may lead to that receptor being activated for long periods. Thus use of nanoparticles may prove to be a very efficient method of delivering a drug to a cell. There are two possible events, the one usually reported is the endocytosis of the particles after preliminary capture at the surface of the cell. The other, discovered only recently is that if certain coating proteins are attached to the particle endocytosis is prevented. This requires that the proteins be bound by surface receptors. This is not in itself a disadvantage in many situations as a very large number of drug receptors are located at the cell surface and since one of them can be chosen to bind the particle to a surface receptor irreversibly and another can be attached to the first to be the active drug which will attach to a nearby second receptor. The advantage of this system is that endocytosis does not occur (see Fig 1b) and there is at least the possibility that use of a competing soluble ligand may allow removal of the signal.

So in terms of drug delivery magnetic nanoparticles offer the possibility of use of external magnetic fields to obtain better localisation than could be achieved with non-magnetic particles. But

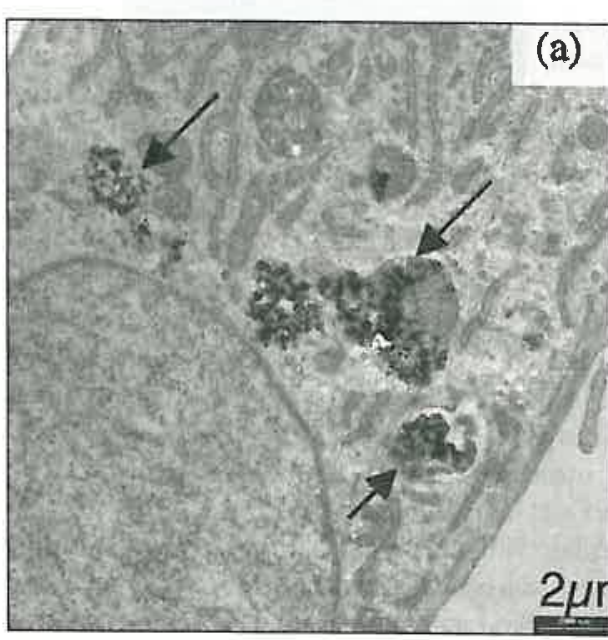

Fig. 1a:

Transmission electron micrograph section (TEM) of a cell (h-tert human fibroblast) that has endocytosed uncoated magnetite nanoparticles, Note their location in the phagolyzosome, arrows indicate phagolysosomes. 
since magnetic nanoparticles are less easily destroyed or inactivated by cells than many non-magentic ones there is the disadvantage that persistent particles may cause later cell damage and death. The same considerations apply to situations where magnetic nanoparticles are being used for generating hyperthermia by the application of external fields.

Cartmell et al. (2003) have suggested that mechanical stimulation could be applied to cells by using an external magnetic field to apply a twisting motion to the particles. Since mechanical stimulation is effective in activating cells this may be a future area for therapeutic developments.

One interesting if rather worrying possi-

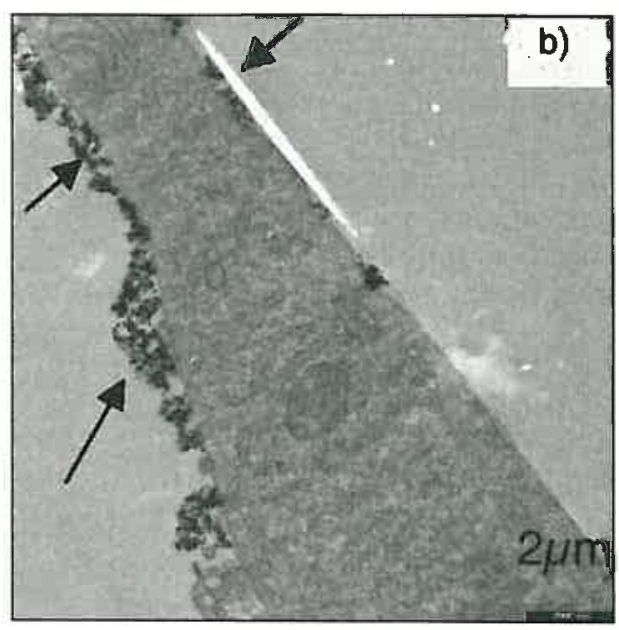

b) bility is that exposure of cells to nanofeatures or nanoparticles can cause large, if perhaps only temporary, changes in gene expression. This would not necessarily be linked solely to the use of magnetic nanoparticles because the work of Dalby et al. (2003) shows that nanofeatures on polymer surfaces can cause change in gene expression.

Since excess particles may well be adsorbed onto the surfaces of cells and intercellular material it is appropriate to consider how these adsorbed particles might affect cell behaviour. This has hardly been examined but some recent work (Berry and Curtis 2003) suggests that they may act much as nanofeatured surfaces do.

\section{References}

Berry, C and Curtis A. (2003) Applications of Magnetic Nanoparticles in Biomedicine

Journal of Physics D:Applied Physics
Cartmell, S.H. et al. (2003) Development of magnetic particle techniques for long term culture of bone cells with intermittent mechanical stimulation. IEEE Transactions in Nanobioscience 1.92-97

Cranfield, Cet al. (in press) IEEE Transactions in Nanobioscience Dalby, M.J., et al.(2003). Interaction of human and tissue cell types with 95nm high nanotopography. IEEE Transactions in Nanobioscience 1:18-23 Kobayashi, H., et al. (2003). Micro-magnetic resonance lymphangiography in mice using a novel dendrimer-based magnetic resonance imaging contrast agent. Cancer Research 63(2): 271-276

Poduslo, J.F. et al. (2002). Molecular targeting of Alzheimer's amyloid plaques for contrast-enhanced magnetic resonance imaging Neurobiology of Disease 11, 315-329

The J. Magnetism and Magnetic Materials Volume 194, Issue 1-3, April 1999 published about 40 papers relevant to this area. A title list can be found at http://www.infomag.ru:8082/dbase/J087E/010503-101.txt 